\title{
A NOTE ON THE ONSAGER MODEL OF NEMATIC PHASE TRANSITIONS *
}

\author{
I. FATKULLIN ${ }^{\dagger}$ AND V. SLASTIKOV $\ddagger$
}

\begin{abstract}
We study Onsager's free energy functional for nematic liquid crystals with an orientation parameter on a unit circle. For a class of interaction potentials we obtain explicit expressions for all critical points, analyze their stability, and construct the corresponding bifurcation diagram. We also derive asymptotic expansions of the equilibrium density of orientations near the critical and zero temperatures.
\end{abstract}

Key words. nematic phase transitions, Onsager model, Maier-Saupe potential.

AMS subject classifications. 82B27, 82D60

\section{Introduction}

In his seminal paper [8] Onsager introduced a simple model describing nematic phase transitions of rod-like polymers. His theory is based on the second-order virial approximation of the free energy as a functional of density of rod orientations. Under a suitable variational ansatz, he was able to demonstrate that at sufficiently high concentrations, rod-like polymers form a nematic phase.

In this note we discuss Onsager's model with an orientation parameter on a unit circle. The corresponding free energy functional reads,

$$
\mathcal{F}[\rho]:=\int_{0}^{2 \pi}\left[\tau \rho(\phi) \ln \rho(\phi)+\frac{1}{2} \rho(\phi) \int_{0}^{2 \pi} U(\phi-\psi) \rho(\psi) \mathrm{d} \psi\right] \mathrm{d} \phi .
$$

Here $\rho(\phi)$ is a probability density of rod orientations ( $\rho \geq 0$ with total integral one). We assume that the interaction potential $U(\phi)$ is given by,

$$
U(\phi)=-\cos n \phi, \quad n=1,2, \ldots
$$

The generally accepted Maier-Saupe potential [7, 5] corresponds to $n=2$; however, since our method is equally applicable to any $n$, we leave it as a parameter. Here we fix the strength of the interaction and vary the temperature $\tau$. We prove that for $\tau>\tau_{c}=1 / 2$ there exists only one critical point (the global minimizer) of $\mathcal{F}$ the uniform density $\bar{\rho}=1 / 2 \pi$. This corresponds to the disordered phase. For $\tau<\tau_{c}$ the uniform density loses stability, and a family of rotation-equivalent critical points, given by,

$$
\rho_{\phi_{0}}(\phi)=\mathcal{Z}^{-1} \mathrm{e}^{\beta(\tau) \cos n\left(\phi-\phi_{0}\right)}
$$

emerges. Here $\phi_{0}$ is an arbitrary parameter and the function $\beta(\tau)$ is obtained by inverting (2.10). These critical points become the global (non-isolated) minimizers and correspond to the ordered nematic phase. No other critical points exist in this system. In addition to this analysis we present asymptotic expansions of $\rho_{\phi_{0}}(\phi)$ as $\tau \downarrow 0$ and $\tau \uparrow \tau_{c}$, and discuss possible generalizations of our method.

Similar results have been obtained in the recent work of P. Constantin, I. G. Kevrekidis, and E. S. Titi [2,3]; and of C. Luo, H. Zhang, and P. Zhang [6].

${ }^{*}$ Received: October 26, 2004; accepted (in revised version): November 22, 2004. Communicated by Weinan E.

${ }^{\dagger}$ California Institute of Technology, USA (ibrahim@acm.caltech.edu).

$\ddagger$ Carnegie Mellon University, USA (slastiko@andrew.cmu.edu). 
Both papers only consider the case $n=2$. Constantin et al., obtain the general form of solutions (though in a different way), but do not make a precise statement about the number of critical states (after submission of this manuscript it was communicated to us by P. Constantin, that a simple way to account for all critical points was also reported in [4]). C. Luo et al., analyze the structure of critical points in Fourier representation, obtain the critical temperature $\tau_{c}$, and classify all critical points. Our method is more transparent and allows us to obtain explicit expressions for all critical points. We hope that this note (our work has been completed independently of both groups) has valuable methodological insights.

2. Euler-Lagrange equation and explicit expressions for critical points The Euler-Lagrange equation for critical points of the free energy (1.1) reads

$$
\int_{0}^{2 \pi} U(\phi-\psi) \rho(\psi) \mathrm{d} \psi+\tau \ln \rho(\phi)=\mu,
$$

where $\mu$ is the Lagrange multiplier which assures that the integral of $\rho$ is unity. Let us introduce the thermodynamic potential $\Phi$ by relation

$$
\rho(\phi)=: \mathcal{Z}^{-1} \exp \{-\Phi(\phi)\}, \quad \mathcal{Z}=\int_{0}^{2 \pi} \exp \{-\Phi(\phi)\} \mathrm{d} \phi .
$$

Note that both $\rho$ and $\Phi$ implicitly depend on the temperature $\tau$. The Euler-Lagrange equation (2.1) can be written as

$$
\tau \Phi(\phi)=\int_{0}^{2 \pi} U(\phi-\psi) \rho(\psi) \mathrm{d} \psi
$$

Differentiating this equation twice with respect to $\phi$, we obtain

$$
\partial_{\phi \phi}^{2} \Phi(\phi)=-n^{2} \Phi(\phi) .
$$

Now let us show that any solution of this equation produces a legitimate solution of the Euler-Lagrange equation (2.1) for some temperature $\tau$.

An arbitrary solution of (2.4) is given by $\Phi(\phi)=-\beta \cos n\left(\phi-\phi_{0}\right)$ for some $\beta \geq 0$ and $\phi_{0}$ (shifting $\phi_{0}$ by $\pi / n$ changes the sign of $\beta$ ). Define a candidate solution of $(2.1), \varrho(\phi)$, by

$$
\varrho(\phi):=\mathcal{Z}^{-1} \mathrm{e}^{\beta \cos n\left(\phi-\phi_{0}\right)}, \quad \mathcal{Z}=\int_{0}^{2 \pi} \mathrm{e}^{\beta \cos n \psi} \mathrm{d} \psi
$$

Observe that $\beta=0$ generates the uniform density $\varrho=\bar{\rho}$,

$$
\bar{\rho}(\phi)=\frac{1}{2 \pi}
$$

which solves (2.1) for all temperatures $\tau$. From now on we will assume $\beta>0$. Let us verify that any $\varrho$ given by (2.5) satisfies the Euler-Lagrange equation (2.1) for some $\tau$. Substituting $\varrho$ and $\Phi$ into (2.3) and recollecting (1.2), we obtain that

$$
\beta \tau \cos n\left(\phi-\phi_{0}\right)=-\int_{0}^{2 \pi} U(\phi-\psi) \varrho(\psi) \mathrm{d} \psi=\mathcal{Z}^{-1} \int_{0}^{2 \pi} \cos n(\phi-\psi) \mathrm{e}^{\beta \cos n\left(\psi-\phi_{0}\right)} \mathrm{d} \psi
$$


should hold for all $\phi$ and some constant (independent on $\phi$ ) $\tau$. Using

$$
\cos n(\phi-\psi)=\cos n\left(\phi-\phi_{0}\right) \cos n\left(\psi-\phi_{0}\right)+\sin n\left(\phi-\phi_{0}\right) \sin n\left(\psi-\phi_{0}\right)
$$

and integrating (the term with $\sin n\left(\phi-\phi_{0}\right)$ disappears) we get

$$
\beta \tau \cos n\left(\phi-\phi_{0}\right)=\mathcal{Z}^{-1} \cos n\left(\phi-\phi_{0}\right) \int_{0}^{2 \pi} \cos n \psi \mathrm{e}^{\beta \cos n \psi} \mathrm{d} \psi
$$

The fact that dependence on $\phi$ appears on both sides only through $\cos n\left(\phi-\phi_{0}\right)$ guarantees that $\tau$ (if it exists for given $\beta$ ) does not depend on $\phi$, as required. Using the definition of $\mathcal{Z},(2.5)$, we can rewrite (2.8) as,

$$
\tau=\frac{1}{\beta} \frac{\mathrm{d}}{\mathrm{d} \beta} \ln \mathcal{Z}=\frac{1}{\beta} \frac{\mathrm{d}}{\mathrm{d} \beta} \ln \int_{0}^{2 \pi} \mathrm{e}^{\beta \cos n \psi} \mathrm{d} \psi .
$$

This integral can be computed employing Sommerfeld's representation for Bessel functions [1], and we readily obtain,

$$
\tau=\frac{1}{\beta} \frac{\mathrm{d}}{\mathrm{d} \beta} \ln \mathrm{I}_{0}(\beta)=\frac{1}{\beta} \frac{\mathrm{I}_{1}(\beta)}{\mathrm{I}_{0}(\beta)}
$$

The function on the right-hand side is symmetric and positive, thus this equation has a positive solution $\tau(\beta)$ for any $\beta$. We conclude that every solution of $(2.4)$ induces a proper solution of the Euler-Lagrange equation (2.1). In order to relate the temperature $\tau$ in (2.1) to solutions of (2.4), we have to find all values of $\tau$, such that equation (2.10) can be resolved for $\beta$. Let us show that $\tau(\beta)$ is a positive bell-like function of $\beta$ with unique extremum (maximum) at $\beta=0$, as displayed on Fig. 2.1. For this we prove that $\beta=0$ is the only root of $\tau^{\prime}(\beta)=0$. After some manipulations with Bessel functions we find

$$
\frac{\mathrm{d} \tau}{\mathrm{d} \beta}=\frac{A(\beta)}{2 \beta \mathrm{I}_{0}^{2}(\beta)}, \quad A(\beta)=\mathrm{I}_{0}(\beta) \mathrm{I}_{2}(\beta)-\mathrm{I}_{1}^{2}(\beta)
$$

$A^{\prime}=\mathrm{I}_{0}\left(\mathrm{I}_{3}-\mathrm{I}_{1}\right) / 2$, thus $A(\beta) \leq 0$, equality is only achieved at $\beta=0$. In the limit as $\beta \rightarrow 0, A(\beta)=\mathcal{O}\left(\beta^{2}\right)$, thus the only root of $\tau^{\prime}(\beta)=0$ is zero and the function $\tau(\beta)$ is monotone-decreasing for $\beta>0 . \tau(0)=1 / 2$ and as $\beta \rightarrow \infty, \tau \downarrow 0$, therefore the inverse function $\beta(\tau)$ has domain $(0,1 / 2]$ within which it has a unique positive branch.

We summarize that for $\tau \geq \tau_{c}:=1 / 2$ the only solution of $(2.1)$ is the uniform density $\bar{\rho}(\phi)$ given by (2.6). For $\tau<\tau_{c}$, except $\bar{\rho}$, there exists a family (parameterized by $\left.\phi_{0}\right)$ of rotation-equivalent solutions

$$
\rho_{\phi_{0}}(\phi):=\mathcal{Z}^{-1} \mathrm{e}^{\beta(\tau) \cos n\left(\phi-\phi_{0}\right)}, \quad \mathcal{Z}=\int_{0}^{2 \pi} \mathrm{e}^{\beta(\tau) \cos n \psi} \mathrm{d} \psi,
$$

where $\beta(\tau)$ is a unique positive solution of (2.10) (recollect that negative $\beta$ can be obtained shifting $\phi_{0}$ by $\pi / n$ and is accounted for by freedom in choosing $\left.\phi_{0}\right)$. Observe that solutions $\rho_{\phi_{0}}(\phi)$ are $n$-periodic: $\rho_{\phi_{0}}(\phi+2 \pi / n)=\rho_{\phi_{0}}(\phi)$, different solutions are only obtained for $\phi_{0} \in[0,2 \pi / n)$. Note also that $\tau_{c}$ does not depend on $n$, i. e., the number of wells in the potential $U(\phi)$. 


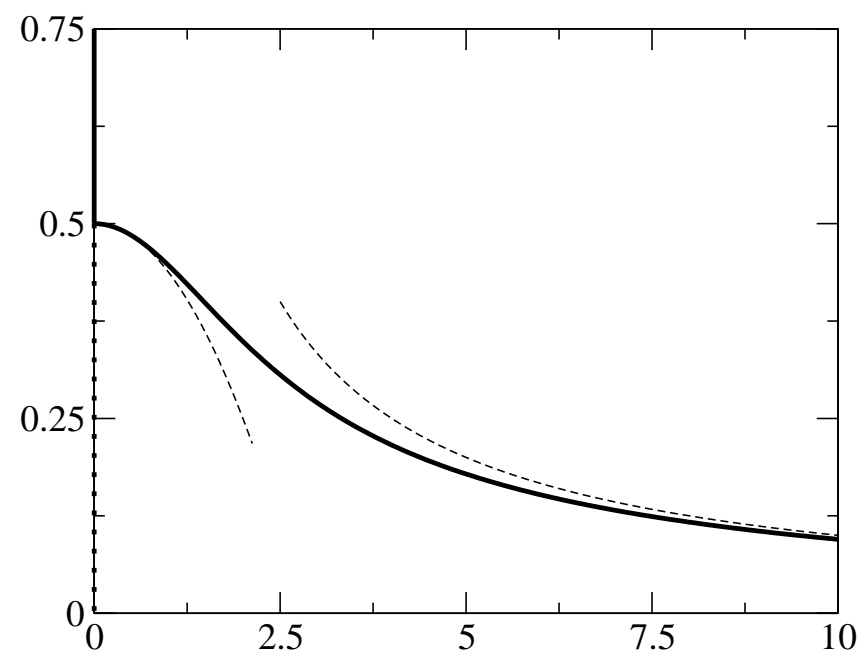

FIG. 2.1.

The bell-shaped curve is the graph of $\tau(\beta)$, given by (2.10); this function is symmetric so only $\beta \geq 0$ is displayed. The dashed lines represent asymptotic expansions as given by (4.1) and (4.2). The thick solid line marks the stable branches: for $\tau>1 / 2$ the uniform density $\bar{\rho}=1 / 2 \pi$ is the the global minimizer, which corresponds to $\beta=0$. It loses stability for $\tau<1 / 2$ (marked by thick dotted line) and $\rho_{\phi_{0}}(\phi)$ become the global minimizers.

\section{Stability of critical points}

To determine stability of the uniform density $\bar{\rho}=1 / 2 \pi$, we compute the second variation of the free energy functional:

$$
\mathbf{D}^{2} \mathcal{F}_{\mid \bar{\rho}}[\eta]=2 \pi \tau \int_{0}^{2 \pi} \eta^{2}(\phi) \mathrm{d} \phi-\frac{1}{2} \iint_{0}^{2 \pi} \cos n(\phi-\eta) \eta(\phi) \eta(\psi) \mathrm{d} \psi \mathrm{d} \phi
$$

In Fourier coordinates this reads,

$$
\mathbf{D}^{2} \mathcal{F}_{\mid \bar{\rho}}[\eta]=-2 \pi\left|\hat{\eta}_{n}\right|^{2}+4 \pi \tau \sum_{k=1}^{\infty}\left|\hat{\eta}_{k}\right|^{2}
$$

We used the fact that $\hat{\eta}_{0}=0$, since $\rho$ is a probability density. From (3.2) we see that the second variation of $\mathcal{F}$ is positive for any $\eta \not \equiv 0$ if and only if $\tau>1 / 2=\tau_{c}$. Thus the uniform density $\bar{\rho}$ is the global (isolated) minimizer for $\tau>\tau_{c}$.

For $\tau<\tau_{c}$ the uniform density $\bar{\rho}$ loses stability. At the same time for $\tau<\tau_{c}$ we have a family of critical points $\rho_{\phi_{0}}(\phi)$ (which all lie on a continuous curve parameterized by $\phi_{0}$, and have the same energy). Since minimizers of $\mathcal{F}$ exist for any positive $\tau$, and $\rho_{\phi_{0}}$ are the only other critical points, we conclude that the latter become global (nonisolated) minimizers. At $\tau=\tau_{c}$ we have a unique critical point $\bar{\rho}$, which is therefore the global minimizer.

The bifurcation diagram corresponding to the transfer of stability from $\bar{\rho}$ to $\rho_{\phi_{0}}$ at $\tau=\tau_{c}$ is also displayed on Fig. 2.1. The thick solid line corresponds to the stable branches: $\bar{\rho}$ for $\tau>\tau_{c}$, and $\rho_{\phi_{0}}$ for $\tau<\tau_{c}$. The thick dotted line corresponds to the uniform density at $\tau<\tau_{c}$, when it becomes a saddle point. Thus the bifurcation which 
occurs in this system can be classified as pitchfork: at $\tau=\tau_{c}$ stability is transferred from a stable node, which then becomes a saddle, to the family of rotation-equivalent stable points. Note that each of $\rho_{\phi_{0}}$ is stable, but not asymptotically stable, since they are all connected by a continuous curve.

\section{Asymptotic expansions}

Having an explicit expression (2.10) and using the well-known asymptotic expansions for Bessel functions [1], we can readily obtain asymptotic expansions of $\beta(\tau)$ as $\tau \downarrow 0$ or $\tau \uparrow \tau_{c}$. We will limit ourselves to the leading terms only. Near $\tau=0$ we obtain, as expected,

$$
\beta(\tau)=\frac{1}{\tau}+\mathcal{O}(1), \quad \text { as } \quad \tau \downarrow 0 .
$$

Expansion near $\tau=\tau_{c}=1 / 2$, corresponds to $\beta \rightarrow 0$. We get

$$
\beta^{4}(\tau)=16\left(\tau_{c}-\tau\right)+\mathcal{O}\left(\tau_{c}-\tau\right)^{2}, \quad \text { as } \quad \tau \uparrow \tau_{c} .
$$

These approximate expressions are displayed on Fig. 2.1 together with the exact solution and demonstrate a reasonable match even in the leading order. Asymptotic expansions for $\rho_{\phi_{0}}$ are obtained by direct substitution of expansions for $\beta(\tau)$ into (2.12): $\rho_{\phi_{0}}(\phi)$ only depends on $\tau$ through $\beta$.

\section{Discussion}

Let us summarize that we have obtained a complete classification of all critical points of the free energy functional (1.1) and have given their explicit representation (2.12). The first crucial step in our approach is transition from (2.3) to (2.4). It is based on the observation that if the interaction potential $U(\phi, \psi)$ satisfies a linear differential equation $\hat{\mathcal{L}} U(\phi)=0$, then the thermodynamic potential $\Phi(\phi)$ necessarily satisfies the same equation. This observation remains valid if the orientation parameter $\phi$ belongs to a higher-dimensional sphere, e. g., $\mathbb{S}^{2}$, and the potential $U(\phi, \psi)$ is invariant under the respective symmetry group. In this case $\hat{\mathcal{L}}$ is the Laplace-Beltrami (or a more general, rotation invariant) operator on the unit sphere.

The next crucial step is to determine when solutions of $\hat{\mathcal{L}} \Phi=0$ generate legitimate solutions of the original Euler-Lagrange equation. In our case this step corresponds to the observation that in (2.8) dependence on $\phi$ appears on both sides only through $\cos n\left(\phi-\phi_{0}\right)$. Generally, one has to choose such solutions $\Phi(\phi)$, that certain solvability conditions are satisfied. Although for a class of potentials considered here it comes out "for free", it is the most nontrivial step in generalization of our approach: it assures existence of temperature $\tau$ (which should be independent of $\phi$ ).

Acknowledgement: The authors thank Weinan E of Princeton University for pointing out that similar results have been obtained in [6], which was not available in press when this work was completed. V. S. acknowledges support by NSF grant DMS-0405343.

\section{REFERENCES}

[1] Abramowitz M. and Stegun I. A., Handbook of Mathematical Functions, with Formulas, Graphs, and Mathematical Tables, Dover Publications, 1974.

[2] Constantin P., Kevrekidis I. G. and Titi E. S., Asymptotic states of a Smoluchowski equation, archive for Rational Mech. and Anal., 174, 3, 365-384, 2004.

[3] Constantin P., Kevrekidis I. G. and Titi E. S., Remarks on a Smoluchowski equation, Discrete and Continuous Dynamical Systems 11, 1, 101-112, 2004. 
[4] Constantin P. and Vukadinovic J., Note on the number of steady states for a $2 D$ Smoluchowski equation, Nonlinearity, 18, 1, 441-443, 2005.

[5] Doi M. and Edwards S. F., The Theory of Polymer Dynamics, Oxford University Press, 1986.

[6] Luo C., Zhang H. and Zhang P., The structure of equilibrium solutions of the one-dimensional Doi equation, Nonlinearity, 18, 379-389, 2005.

[7] Maier W. and Saupe A., Eine einfache molekulare Theorie des nematischen kristallinflüssigen Zustandes, Zeitschrift für Naturforschung A, 13, 564, 1958.

[8] Onsager L., The effects of shape on the interaction of colloidal particles, Annals of New York Academy of Sciences, 51, 627-659, 1949. 